\title{
Graphical and Numerical Diagnostic Tools to Assess Suitability of Multiple Imputations and Imputation Models
}

\section{Bondarenko and T. E. Raghunathan*}

Multiple imputation has become a popular approach for analyzing incomplete data. Many software packages are available to multiply impute the missing values and to analyze the resulting completed data sets. However, diagnostic tools to check the validity of the imputations are limited and the majority of the currently available methods need considerable knowledge of the imputation model. In many practical settings, however, the imputer and the analyst may be different individuals or from different organizations, and the analyst model may or may not be congenial to the model used by the imputer. This article develops and evaluates a set of graphical and numerical diagnostic tools for two practical purposes: (1) For an analyst to determine whether the imputations are reasonable under his/her model assumptions without actually knowing the imputation model assumptions; and (2) For an imputer to fine tune the imputation model by checking the key characteristics of the observed and imputed values. The tools are based on the numerical and graphical comparisons of the distributions of the observed and imputed values conditional on the propensity of response. The methodology is illustrated using simulated data sets created under a variety of scenarios. The examples focus on continuous and binary variables but the principles can be used to extend methods for other types of variables.

Copyright $(\mathbf{c} 0000$ John Wiley \& Sons, Ltd.

Keywords: multiple imputation; propensity score; diagnostics; congeniality

\section{Introduction}

Multiple Imputation (MI) is a general purpose approach for analyzing data with missing values where the missing set of values are replaced by several sets of plausible values. These values are generated as draws, typically from a predictive distribution of the missing set conditional on the observed set of values. Each plausible or imputed set, when combined with the observed set of values, results in a completed data set. Each completed data set is then analyzed separately and then the inferential statistics (such as point estimates, covariance matrices, test statistics or p-values) are combined across

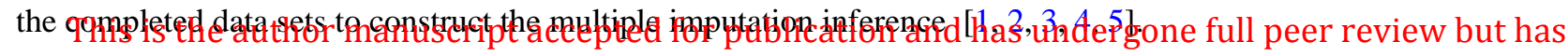

not been through the copyediting, typesetting, pagination and proofreading process, which may lead to differences between this version and the Version of Record. Please cite this article as doi: 


\section{Statistics}

Data with missing values may be complex with several types of variables, (such as continuous, ordinal, nominal, count, semi-continuous, etc.), involve skip patterns (for example, some variables are not applicable to a particular group of subjects), and restrictions (such as bounds for plausible lab values). Availability of multiple imputation software to handle such complexities has made the multiple imputation procedure attractive. During the last two decades, procedures for multiple imputation of missing values have been incorporated into many popular statistical software packages such as SAS, R, Stata $[6,7,8,9]$.

Despite this extensive development of imputation software, the tools to diagnose the validity of imputations are limited, and the majority of currently available tools assume that the imputation model is known. In most practical applications of multiple imputations, however, the imputer and the analyst are different individuals or may work at different organizations. Hence the analyst may have limited or no knowledge of the imputation model. This may lead to a scenario where the imputation and analyst models make different assumptions with respect to data generating process and, hence, in broad terms uncongenial [10].

There are different kinds of uncongeniality having varying consequences on the point and interval estimates. For example, if the analyst model is a submodel of the imputer model then the point estimates are typically unbiased but may yield wider interval estimates. On the other hand, if the imputer model is a submodel of the analyst model then the point estimates may be biased. Another type of uncongeniality occurs when the model assumptions made by the imputer and analyst are the same but the analyst uses suboptimal estimation procedure (for example, the method of moments instead of the maximum likelihood) $[11,12]$. Note, that we always assume that the analyst model is the correct model for performing the repeated sampling calculations.

This paper develops a set of diagnostic tools with two broad objectives:

1. To assist an analyst to determine whether the imputations are reasonable under his/her model, without actually knowing the exact imputation model. That is, the analyst can diagnose uncongeniality.

2. To assist an imputer to fine tune the imputation model through checking whether the observed and imputed values exhibit similar characteristics, and thus capturing important features to be preserved in the imputation process.

An imputer can use several standard model building techniques such as regression diagnostics, posterior predictive checks, variable selection methods etc. These tools allow the modeler to discern important features and structures in the data and then to incorporate them in the imputation model. Some examples include inclusion of nonlinear or interaction terms, variable transformation etc. The goal of the second objective listed above is slightly different from these standard procedures. That is, the goal is to check whether, conditional on the model chosen, the observed and imputed values exhibit similar characteristics. After all, the goal of the imputation is to create a plausible completed data set from the population. If the observed and imputed values are not exhibiting similar characteristics, then the imputation model needs further refinement.

Recently, several imputation diagnostic tools have been proposed $[13,14,15,16]$. These tools proceed in two stages: (1) Initial comparison of the marginal distributions of the imputed and observed values; ( 2) Evaluation of variables flagged based on the first stage with the knowledge of the imputation model. The initial comparison of the marginal distributions of the observed and imputed values is useful to identify variables that need future evaluation. Existing statistical packages provide a variety of strategies for comparison of marginal distributions of imputed and observed values, including comparison of histograms, density plots, $q-q$ plots, or descriptive statistics of the imputed and observed. Various numeric tests and rules of thumb have been suggested to identify variables with differences in the marginal distributions of observed and imputed values. For example, flagging variables with an absolute difference in means between the observed and imputed values greater than 2 standard deviations, or with a ratio of variances of the observed and imputed values that is less than 0.5 or greater than 2 [16]. As an alternative, compare variances of point estimates and correlation coefficients from multiply imputed data and available cases [14]. Kolmogorov-Smirnov test can also be used to flag variables with significant differences in the marginal distributions of imputed and observed values [13]. In such 


\section{Statistics}

comparisons attention should be paid to the extreme values produced by imputations, because they can indicate a potential problem with imputations and a need for model improvement [15].

It is important to note that the marginal distributions of the observed and imputed values are expected to be similar only when the data are missing completely at random (MCAR). Under the missing at random mechanism (MAR), the differences in the marginal distributions of the observed and imputed values is not necessarily an indicative of problems with the imputation model. Consider a bivariate example with missing values in $Y$ with $X$ fully observed. Suppose that the probability of missing $Y$ values is positively (or negatively) correlated with $X$. Depending upon the correlation between $Y$ and $X$, the marginal distributions of the observed and imputed values will differ even when the imputations are created under the correctly specified model. Thus, the initial evaluation described above may result in false alarms about the imputation model. Thus, many of these procedures are mostly useful for filtering a large set to smaller subset for further inspection.

The second stage of MI diagnostics includes more elaborate tools developed to assist in the evaluation of certain conditional, weighted distributions of observed and imputed values. For example, one can compare complete-case, MI , and weighted analyses [14]. This approach, however, is applicable only for a certain missing data patterns. An alternative is to test the fit of the imputation model to the observed data by comparing distributions of the residuals for imputed and observed values, conditional on their predicted values under the imputation model [13]. If the imputation model is a good fit, then the pattern of residuals should be random with no difference between the observed and imputed values. A sensitivity analysis based on the posterior predictive checking under an imputation model or its surrogate based on the subset of variables may also be useful [14]. For example, suppose that the completed data through imputation, $D_{C}$, is used to generate several copies or replicates $D_{C}^{*(r)}, r=1,2, \ldots$ through posterior predictive check mechanism. If the original imputations are valid, then the estimates based on the completed data through imputation, $D_{C}$, should be similar to the estimates calculated from $D_{C}^{*(r)}$ (under the correctly specified imputation model). However, considerable knowledge of the imputation model is necessary to implement many of these methods.

As emphasized earlier, the imputation models may not be available when the analyst and imputer work in different organizations. Sometimes, internal variables (such as paradata or administrative data) may be used in the imputation process which may not be released as a part of the completed data sets. In such situations, it is necessary to determine whether the imputations are valid under the analyst model or, in other words, to assess whether the imputation model is congenial with the model posited by the analyst. The notion of congeniality of the imputation and analyst models is an important issue. The techniques proposed in this paper can be used to assess congeniality of imputations for the analyst model without knowing the imputation model.

The rest of the paper is organized into the following five sections. Section 2 develops the proposed diagnostic methods. Section 3 outlines a set of graphical tools to implement the method for continuous and binary variables. Section 4 uses simulated data sets to illustrate and evaluate the proposed approaches for assessing the validity of imputations. How these tools can be used for detecting uncongeniality is also discussed in Section 4. Section 5 proposes numerical summaries or test procedures and evaluates them using simulated data sets. Section 6 concludes with discussions, limitations and future work. Throughout the paper, the missing data are assumed to be missing at random.

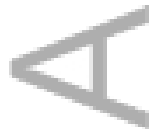

\section{Proposed Method}

Consider a data set with missing values that has $n$ observations and $p$ variables, $Y_{v}, v=1,2, \ldots, p$ and the goal is to assess imputation for the variable $Y_{v}$. Without loss of generality, denote the set of observed values of $Y_{v}$ as $y_{o b s, v}=\left\{y_{s v}, s=\right.$ $\left.1,2, \ldots, n_{v}\right\}$ and the set of missing values as $y_{m i s, v}=\left\{y_{s v}, s=n_{v}+1, n_{v}+2, \ldots, n\right\}$. Let $R_{s v}=1, s=1,2, \ldots, n_{v}$ and $R_{s v}=0, s=n_{v}+1, n_{v}+2, \ldots, n$ be the response indicator. Let $Y_{o b s,-v}$ denote all the observed set of values of variables $Y_{i}, i=1,2, \ldots, v-1, v+1, \ldots, p$ across all subjects. If values of $Y_{v}$ are missing at random then, by 


\section{Statistics}

definition, the conditional distribution of $y_{o b s, v}$ given $Y_{o b s,-v}$ should be the same to that of $y_{m i s, v}$ given $Y_{o b s,-v}$. That is, $\operatorname{Pr}\left(y_{o b s, v} \mid Y_{o b s,-v}\right)=\operatorname{Pr}\left(y_{m i s, v} \mid Y_{o b s,-v}\right)$

Let $e_{o b s,-v}=\operatorname{Pr}\left(R_{v}=1 \mid Y_{o b s,-v}\right)$ be the actual response propensity for variable $Y_{v}$ as a function of the observed data, $Y_{o b s,-v}$. The propensity scores $e_{o b s,-v}$ is an efficient summary of the covariates $Y_{o b s,-v}$ [17].Thus, under the MAR assumption, distributions of observed $y_{o b s, v}$ and missing $y_{m i s, v}$ must be the same, conditional on $e_{o b s,-v}$.

Missing values $y_{m i s, v}$ are unknown quantities and replaced in the course of imputations with a set of imputed values, $y_{m i s, v}^{(l)}=\left\{y_{s v}^{(l)}, s=n_{v}+1, n_{v}+2, \ldots, n\right\}$, where $l=1,2, \ldots, M$. If the imputations are reasonable under the missing at random assumption, then the observed set $y_{o b s, v}$ and the imputed set $y_{m i s, v}^{(l)}$ should have similar distributions conditional on the propensity score $e_{o b s,-v}$ or equivalently, $\operatorname{Pr}\left(y_{o b s, v} \mid e_{o b s,-v}\right) \sim \operatorname{Pr}\left(y_{m i s, v}^{(l)} \mid e_{o b s,-v}\right)$

The actual response propensity, $e_{o b s,-v}$ is not known but it can be estimated for each subject. One option is to estimate the propensity scores in the presence of missing data by conditioning on the response indicators as well as the observed covariates [18]. The second option is to use the following approach to estimate $e_{o b s,-v}$.

1. Let $Y_{m i s,-v}^{(l)}, l=1,2, \ldots, M$ denote the $M$ sets of imputed values for the missing values in all the variables except $Y_{v}$. Analyze each completed data set to build a response propensity model for $Y_{v}$ using the standard logistie, probit or any other regression model with binary outcome variable, $R_{v}$, and the completed data $Y_{i}, i=$ $1,2, \ldots, v-1, v+1, \ldots, p$ as predictors. One could also use nonparametric regression models, such as CART or semiparametric models such as generalized additive models, to estimate the observed data propensity scores. The choice of the model for propensity of response is guided by the best fit model that ensures balance of all covariates, i.e. $\operatorname{Pr}\left(Y_{o b s,-v} \mid e_{o b s,-v}, R_{v}=1\right)=\operatorname{Pr}\left(Y_{o b s,-v} \mid e_{o b s,-v}, R_{v}=0\right)$. The extent of balancing can be determined using the methods described in $[19,20]$. Standard model building tools can be used to inspect whether interaction terms or transformations are needed and to assess the goodness of fit statistics (for example, in Hosmer and Lemeshaw [21]). After a satisfactory fit of the model in each completed data is achieved, obtain the values of the estimated propensities, $\widehat{\operatorname{Pr}}\left(R_{v}=1 \mid Y_{o b s,-v}, Y_{m i s,-v}^{(l)}\right)$.

2. For sufficiently large $M$, approximate

$$
\widehat{e}_{o b s,-v}=\widehat{\operatorname{Pr}}\left(R_{v}=1 \mid Y_{o b s,-v}\right) \approx \sum_{l=1}^{M} \widehat{\operatorname{Pr}}\left(R_{v}=1 \mid Y_{o b s,-v}, Y_{m i s,-v}^{(l)}\right) / M .
$$

\section{Specific Diagnostics}

\subsection{Continuous Variables}

The next step of comparing the imputed and observed values for a variable $Y_{v}$, conditional on the estimated propensity scores, can be performed in a number of ways. For example, $H$ strata could be created from the propensity scores with $n_{h}$ and $m_{h}$ observed and imputed values in stratum $h=1,2, \ldots, H$. Analysis of variance technique may be used with stratum ( $H-1$ degrees of freedom), indicator for observed/imputed (1 degree of freedom) and their interactions ( $H-1$ degrees of freedom) as factors. Under the correctly specified imputation model, the mean squares for both the missingness indicator and the interaction effect should be small. A large between-stratum sum of squares indicates significant departure from MCAR assumption. Since the imputed and observed values are correlated, the derivation of the sampling distribution even under the correctly specified imputation model is complex. Hence, it is analytically difficult to define large or small using the significance testing framework. In Section 5, we describe some empirically derived decision rules to reject imputations that are calibrated (the exact and nominal levels are similar) and good power detect problems with the imputation.

Since the goal is to diagnose the problem with the imputations, a set of graphical tools (similar to the residual plots in a regression analysis) might be more useful. Two useful diagnostic plots are described to visualize the differences in the conditional distributions: Scatter Plot Diagnostics and Residual Density Diagnostics. 


\section{Statistics}

Scatter Plot Diagnostics: Plot values of $Y_{v}$ versus the estimated propensity of response $\widehat{e}_{o b s,-v}$. Use different colors or symbols to identify observed and imputed values in $Y_{v}$. Systematic differences in the patterns of scatter across observed and imputed values, for a given value of propensity of response, indicate problems with the imputation model. Adding separate LOESS curves to the scatter for imputed and observed groups may help further to visualize the differences.

Residual Density Diagnostics First, regress $Y_{v}$ on the estimated propensity score $\widehat{e}_{o b s,-v}$. Next, create histograms or kernel density plots of the residuals, separately for the observed and imputed values in $Y_{v}$. Differences in shape as well as the location of the residual densities indicate problems with imputations.

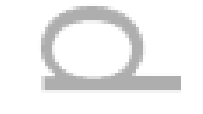

=

\subsection{Diagnostics for Binary Variables}

For a binary variable $Y_{v}$, the analog of ANOVA method, described earlier for the continuous variable, may be developed. As before, create $H$ strata based propensity scores, and fit a logistic regression model with $Y_{v}$ as the outcome (imputed or observed), $H-1$ dummy variables for strata, 1 dummy variable for observed/imputed and their products (interaction). We could use the deviance statistic as a measure and under the correctly specified model, the deviance for both missing indicator and the interaction effect should be small. As in the continuous case, a set of graphical diagnostics tools may be more useful.

TheScatter Plot Diagnostics can be applied to any type of variable: continuous or categorical. For binary variables, the average of the outcome values across imputations gives a better picture (because the response is either 1 or 0 ). Adding LOESS curves plotted on the top of the scatter make this plot, generally, informative. For a categorical variable, we may use the frequency distribution averaged across imputations.

The Residual Density Diagnostic described earlier cannot be directly used for a binary variable because the residuals (given that the observed data is either 1 or 0 ) are not very informative and is even more problematic with nominal categorical outcome. Deviance residuals may be used but the simulation study described in the next section did not show much promise for such residuals.

To develop an analog of Residual Density Diagnostic for a binary variable $Y_{v}$, we modify the method described for the continuous variable as follows. Let $p_{v}$ denote the actual conditional probability $\operatorname{Pr}\left(Y_{v}=1 \mid Y_{-v}\right)$. Then $p_{v}$ is a balancing score for the covariates $Y_{-v}$ across the two populations defined by the outcome $Y_{v}$. In particular, the distributions of $Y_{-v}$, conditional on $p_{v}$ are independent of $Y_{v}$ [17]. This ideas was first proposed in [22] for logistic regression models, and extended to the ordinal outcome in [23].

Note that, as discussed earlier, conditional on $e_{o b s,-v}$, the $Y_{o b s,-v}$ is independent of $R_{v}$. Thus, it follows from the properties of these two scores $e_{o b s,-v}$ and $p_{v}$, that under MAR, conditional on $e_{o b s,-v}$ and $p_{v}$, the $Y_{-v}$ is independent of both $Y_{v}$ and $R_{v}$. That is,

$$
\operatorname{Pr}\left(Y_{-v} \mid e_{o b s,-v}, p_{v}\right) \Perp R_{v}, Y_{v}
$$

A simple proof follows by noting that,

$$
\operatorname{Pr}\left(Y_{-v}, Y_{v}, R_{v} \mid e_{o b s,-v}, p_{v}\right)=\operatorname{Pr}\left(Y_{-v} \mid e_{o b s,-v}, p_{v}\right) \cdot \operatorname{Pr}\left(Y_{v}, R_{v} \mid e_{o b s,-v}, p_{v}, Y_{-v}\right)
$$

Since, under MAR and the property of the propensity scores,

$$
\operatorname{Pr}\left(R_{v}, Y_{v} \mid e_{o b s,-v}, p_{v}, Y_{-v}\right)=\operatorname{Pr}\left(Y_{v} \mid e_{o b s,-v}, p_{v}\right) \cdot f\left(R_{v} \mid e_{o b s,-v}, p_{v}\right),
$$

the right hand side of equation (3.2.1) reduces to

$$
\operatorname{Pr}\left(Y_{-v} \mid e_{o b s,-v}, p_{v}\right) \cdot \operatorname{Pr}\left(Y_{v} \mid e_{o b s,-v}, p_{v}, Y_{-v}\right) \cdot \operatorname{Pr}\left(R_{v} \mid e_{o b s,-v}, p_{v}, Y_{-v}, Y_{v}\right)
$$




\section{Statistics}

The above result implies that the distribution of the covariates conditional on both propensities should be similar for the two populations defined by $Y_{v}$ regardless of whether $Y_{v}$ is imputed or observed. Specifically,

$$
\operatorname{Pr}\left(Y_{-v} \mid e_{o b s,-v}, p_{v}, R_{v}, Y_{v}=0\right) \sim \operatorname{Pr}\left(Y_{-v} \mid e_{o b s,-v}, p_{v}, R_{v}, Y_{v}=1\right)
$$

Note that, matching on both propensity scores is akin to residual density diagnostics for the continuous variable. Estimation of $\widehat{p}_{v}$ poses a challenge because $Y_{v}$ is not observed for $R_{v}=1$, and using the imputed values makes the estimate very much dependent on the imputation model. The suggestion is to estimate $\widehat{p}_{v}$ using $Y_{\text {obs, } v}$ (that is, subset the completed data to $R_{v}=0$ ), conditional on the observed values $Y_{o b s,-v}$. Specifically, fit, for example, a logistic regression (or any other regression) model for

$$
\widehat{p}_{v}^{(l)}=\widehat{\operatorname{Pr}}\left(Y_{v}=1 \mid Y_{o b s,-v}, Y_{m i s,-v}^{(l)}, R_{v}=0\right)
$$

to obtain the maximum likelihood estimate of the regression coefficients, $\widehat{\beta}_{l}$. Note that, the complete-case maximum likelihood estimates are the correct estimates for the regression model under MAR. Average $\widehat{\beta}_{l}$ across imputations $\left(\bar{\beta}=\sum_{l} \widehat{\beta}_{l} / M\right)$ and then apply the resulting logistic regression equation (with the average $\bar{\beta}$ as the regression coefficient) to estimate the propensity score $\widehat{p}_{v}$ for the whole sample.

In the simulation study (described in the next section), the following strategy to develop Residual Density Diagnostic for Binary Variables proved to be highly useful to diagnose problems with the imputations of binary variables. First, conduct a principal component analyses on $Y_{-v}^{(l)}$ and extract the principal components $Z_{-v}^{(l)}$. Next, regress these principal component $Z_{-v}^{(l)}$ on $p_{v}, \widehat{e}_{o b s,-v}$ and their interaction. Finally, separate the data by the missingness indicator $R_{v}$, create two sets of the create histograms or kernel density plots of the residuals, grouping subjects by the value of outcome $Y_{v}$ within each set (a total of four kernel density plots for the cross classification $R_{v} \times Y_{v}$ ). Differences in distributions of the residuals for the two values of $Y_{v}$ for subjects with $R_{v}=1$ or for subjects with missing value of $Y_{v}\left(R_{v}=0\right)$ indicate problem with imputations.

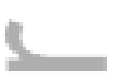

\section{Simulation Study}

To evaluate these diagnostic tools, we conducted a simulation study involving the following four steps: generation of complete data; setting some values to missing; multiply imputing under the correct and incorrect models; and finally applying the diagnostic tools developed in this paper. The simulated data contained both continuous and binary variables.

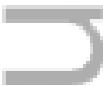

\subsection{Simulations for continuous variables}

First, the complete data of size 1000 were generated from normal model with mean function given by (4.1.1) and variance 1. Variables $x_{1}, x_{2}, x_{3}$ were independently drawn from the standard normal distribution and are assumed to be fully observed.

$$
\mathrm{E}(y \mid x)=\alpha_{0}+\alpha_{1} x_{1}+\alpha_{2} x_{2}+\alpha_{3} x_{3}+\alpha_{4} x_{1}^{2}+\alpha_{5} x_{1} \cdot x_{2} .
$$

We considered two scenarios by choosing the values of $\alpha$. The null scenario or the correctly specified model where $\alpha_{4}=\alpha_{5}=0$, or the non-null scenarios with varying levels of mis-specification by various choices of $\left(\alpha_{3}, \alpha_{4}, \alpha_{5}\right)$. The mis-specification scenarios correspond to omitting a linear, quadratic or interaction term. 


\section{Statistics}

Second, some values for $y$ were deleted. The missing data mechanism was defined by a logistic regression model (4.1.2). The regression coefficients were chosen to yield the response rate as 0.5 (50\% missing values on the average).

$$
\operatorname{logit}(\operatorname{Pr}(y=.))=1-x_{1}+x_{2}-x_{3} .
$$

At the third step the missing values of $y$ were multiply imputed using the sequential regression approach as implemented in IVEWARE [6] package. For this simple case with missing values only in $y$, this is equivalent to drawing values from the posterior predictive distribution under the normal model with non-informative prior for the parameters. The imputation models adopted for these simulations did not include interaction, or a quadratic term (misspecified models but all variables included), and in one scenario did not include $x_{3}$ term (omitted variable in the model). Five multiply imputed data sets were produced under all scenarios.

Lastly, Scatter Plot and Residual Density diagnostic were applied to the imputed data sets to assess the validity of imputations. The results are given in Figure 1. It shows that the two proposed diagnostics and, for comparison purposes, also the expected-value based diagnostics, which requires the knowledge of imputation model [13].

[Figure 1 about here.]

Red and black dots indicate imputed and observed values, respectively. The first row corresponds to the null scenario, where the imputation and data generating models included only linear terms for $x_{1}, x_{2}, x_{3}$ and $y$ with no interactions or square terms. All three methods, as expected, show no difference in patterns of red (imputed) and black (observed) values.

In the second row, the imputation model is misspecified by omitting $x_{3}$. Scatter plot diagnostic shows differences in the observed and imputed values of $y$ for a given values of the propensity scores. The differences in the patterns are made clearer by the addition of LOESS curves to the scatter plot. The residual density plot shows less variance in the residual values for imputed $y$ 's than for the observed values. However, the expected values-based plot shown in the third column fails to diagnose the problem. In fact, if a variable is erroneously omitted from the imputations, the diagnostics based on the imputation model are not valid and hence the expected-value based diagnostic cannot be used to detect omitted variable from the imputation model.

In the third row, the data generating model includes both the linear and quadratic terms for $x_{3}$, but the imputation model includes only a linear term. For this scenario all three methods clearly detect substantial differences in patterns of the observed and imputed values. For example, the residuals for the observed values (in black) show a long left tail, whereas the residuals for the imputed values (in red) show much more compact and symmetric distribution. This difference in in the residual densities indicates a need for a non-linear transformation. We tested this aspect through several replicates of the simulation study.

The final row examines a scenario where the interaction term is a part of data generation model, but not included in the imputation model. Here, the residual density plot and the expected value based diagnostics clearly indicate differences in patterns between observed and imputed values, whereas the scatter plot diagnostics fails to show substantial differences where the response propensities overlap. That is, the residual density and the scatter plot diagnostics call for different conclusions.

A more detailed investigation reveals that the residual density and scatter plot diagnostics target different aspects of misspecification of the imputation model. To investigate further, consider two data sets $A$ and $B$ with the following two regression models for $y$ involving interaction between $x_{1}$ and $x_{2}$ :

$$
\begin{aligned}
& \mathrm{E}(y \mid x)=1+x_{1}+x_{2}+x_{3}+x_{1} \cdot x_{2}, \\
& \mathrm{E}(y \mid x)=1+x_{1}+x_{2}+x_{3}-x_{1} \cdot x_{2},
\end{aligned}
$$

As before, some values of $y$ were set to missing in correspondence using the logistic model (4.1.2) and then multiply imputed both data sets assuming that values of $y$ depend on the linear combination of $x_{1}, x_{2}$, and $x_{3}$ but ignoring the 


\section{Statistics}

\section{in Medicine}

interaction term $x_{1} \cdot x_{2}$.

After the imputations, the marginal and conditional distributions of the original values of $y$ that were set to missing and the corresponding imputed values were compared. The first and second columns of graphs in Figure 2 show the conditional and marginal distributions of the original (red) and imputed (blue) values of $y$. The imputations for data set $A$ (4.1.3) preserved conditional distributions of $y$ given $x_{3}$ but distorted the marginal distribution of the residuals. Imputations of data set $B(4.1 .4)$ retained the marginal distributions of $y$ but distorted the relationship of $y$ and $x_{3}$.

[Figure 2 about here.]

The plots comparing the marginal distributions of the original and imputed values of $y$ are shown in the second and third columns of Figure 2. The proposed diagnostic tools as well as expected-value based diagnostics were applied to check whether they are equally capable to detect flaws in the imputations of data sets A and B. As shown in the last three columns of Figure 2 the scatter plot is effective in recognizing problems with the conditional distributions of imputed values, and the residual density plot targets the marginal distributions of the residuals. If conditional distributions are preserved, but the marginal distributions of the residuals are distorted by imputations (as in data set $A$ ), then the residual density plots and the expected-value based diagnostic are helpful to diagnose the problem. If marginal distributions are similar, but the conditional distributions are distorted (data set $B$ ) then the scatter plots are useful. Thus, it is important to apply both Residual Density Diagnostics and the Scatter plot diagnostics, as they detect different types of misspecfications.

\subsection{Simulations for Binary variable}

The simulation study for a binary variable consisted of the same four steps as for the continuous variable. Binary variable, $y$, was generated by the logistic regression model (4.2.1).

$$
\operatorname{logit}(P(y=1 \mid x))=\alpha_{0}+\alpha_{1} x_{1}+\alpha_{2} x_{2}+\alpha_{3} x_{3}+\alpha_{4} x_{1}^{2}+\alpha_{5} x_{1} \cdot x_{2},
$$

As in the continuous case, values $x_{1}, x_{2}, x_{3}$ are independent draws from standard normal distributions and fully observed. Missing mechanism for $y$ was imposed by the equation (4.1.2).

The values of $y$ were set to be missing and then imputed using IVEWARE. Again, for this simple case, the imputations are the draws from the posterior predictive distribution under the binomial-logistic regression model with non-informative prior for the regression coefficients with the posterior distribution of the parameters being approximated by a multivariate normal distribution with the maximum likelihood estimate as the mean and the inverse of the observed information matrix as the covariance matrix. Imputation models were prone to various degrees of misspecification, including omitted linear, quadratic or interaction terms. The results are illustrated in Figure 3.

[Figure 3 about here.]

The first row corresponds to the correct imputation where the imputation model is consistent with the data generating model. Scatter Plot Diagnostic, in the first column shows observed (black) and imputed (red) values are very similar. Residual Density Diagnostic shows a set of four Kernal Density curves. Again the observed values are depicted by the black lines and the imputed by the red lines. Solid lines of both colors represent observations with $y=0$ and dashed lines represent $y=1$. The kernel densities in the first row are very similar across all four subgroups. Thus, both diagnostics confirm the validity of imputations.

The second row of graphs corresponds to the omitted-variable scenario. LOESS curves are clearly different for the observed and imputed values of $y$. For the third row, a case of ignored interaction, the Scatter Plot Diagnostic clearly diagnoses a problem with imputations. On the other hand, the Residual Density Diagnostic are not very informative.

The last row shows a scenario where a square term for $x_{3}$ is present in the data generation model but is omitted from the imputation model. Here the Scatter Plot Diagnostic is rather ambiguous. LOESS curves for the observed and imputed values of $y$ coincide in the area of the most dense overlap of propensities for respondents and nonrespondents, and differ 


\section{Statistics}

on the sides. However, residual plot clearly shows the difference in distributions for nonrespondents for whom $y$ was imputed to be 0 and for those with $y$ imputed as 1 . The last two scenarios indicate discrepancy between conclusions from Scatter Plot Diagnostic and Residual Density Diagnostic. As for the continuous variable, it is important to check both diagnostic tools to evaluate the imputations.

\subsection{Assessment of Uncongeniality}

An analyst can detect uncongeniality by constructing Scatter Plot Diagnostic and Residual Density Diagnostic using the residuals from the analyst model. Suppose that the analyst using a regression model that involves $Y_{v}$ and has some imputed values. Suppose, that the analyst model was applied to the imputed data set $l$ yielding the residuals $r^{(l)}$. If the imputations are "agreeable" with the analyst model, then under MAR assumption, in each imputed data set $l$ distribution of the residuals $r^{(l)}$, conditional on the estimated propensity of response, must be similar for $R_{v}=1$ and $R_{v}=0$ groups.

To create Residual Density diagnostic first regress $r^{(l)}$ on the propensity score $\widehat{e}_{o b s,-v}$ and construct the residuals $u^{(l)}$ from this model. Next, or each imputation $l$ generate the Kernel Density Plots for $u^{(l)}$ by $R_{v}$. If imputation model is congenial to the analyst model then the marginal distributions of the residuals $u^{(l)}$ must be similar between the two groups, respondents and nonrespondents.

To further elaborate on this approach, we simulated data for the congenial and uncongenial scenarios. Specifically, an analyst is interested in a regression model for $y$ as a linear function of $x_{1}, x_{3}$ and their product. The analyst believes that interaction between $x_{1}$ and $x_{3}$ is important. However, the analyst has no knowledge if the interaction was included in the imputation model and wants to assess if the imputations are valid under the posited model.

We simulated the data under the analyst model, reset some values of $y$ to missing, and then multiply imputed the two sets data. One set was imputed incorporating interaction $x_{1} \cdot x_{3}$ into the imputation model. The second set was imputed ignoring the interaction. The Figure 4a shows the Scatter Plot and Residual Density diagnostics for the congenial models were imputation model included the interaction. Neither plot shows any differences between distributions for the residuals based on the observed and imputed values. However, the plots in Figure $4 b$, corresponding to the imputations omitting the interaction term, show differences between the observed and imputed values. These differences allow the analyst to conclude that imputations are not reasonable under the analyst model.

[Figure 4 about here.]

We conducted several other simulation studies varying the missingness rate, alternative models for both regression model for the outcome as well as missing data mechanism. Across all these simulation studies, the proposed diagnostic procedure detected modest to severe discrepancies. When the residual variance in the outcome regression model is large compared to systematic bias due to underfitting, the residual diagnostic plots were better in detecting the problems than the scatter plot diagnostic. Generally, together they practically identified problems when they exist.

\section{Numerical Tests}

Though the emphasis of this paper has been on graphical approach, we also propose two test procedures that mirror the graphical diagnostic tools. These are useful to formally test the validity of imputations using the significance testing framework.

As indicated earlier, a numeric test analogous to the scatter plot diagnostics can be performed applying the analysis of variance technique. A similar approach has been used to ensure balance of covariates on the estimated propensity score [17] . To apply the ANOVA technique to diagnose problems, the estimated probabilities of response $\widehat{e}_{o b s,-v}$ are grouped into $H$ strata. The analysis of variance model includes stratum $(H-1$ degrees of freedom), indicator for observed/imputed ( 1 degree of freedom) and their interactions ( $H-1$ degrees of freedom) as factors. It has been shown that with five strata 


\section{Statistics}

$90 \%$ of the bias is removed $[19,24]$ Thus, we use $H=5$ as default guidance for this test. The ANOVA test basically uses a F-statistic that compares the full model with the null model that drops the missing data indicator and stratum by indicator interaction term.

As a numerical analog of KDE diagnostics, we propose using the Kolmogorov-Smirnov (KS) test for equality of the distribution of the residuals for the imputed and observed units conditional on the estimated propensity of response. Define the residuals $\boldsymbol{r}_{s v}=y_{s v}-\hat{y}_{s v}$, where $\hat{y}_{s v}$ is the prediction from the regression of $Y_{v}$ on $\widehat{e}_{o b s,-v}$. Compute the KS statistic comparing the residuals for $R_{v}=1$ with $R_{v}=0$. Thus the ANOVA test would formally test the equality of the location, and the Kolmogorov-Smirnov (KS) test examines equality of the full residual distribution.

As indicated earlier, due to correlation between the imputed and observed values, the standard sampling distributions for the ANOVA and KS procedures cannot be applied. It is difficult to analytically derive the actual sampling distributions. Instead we conducted a simulation study by generating data under both null and non-null scenarios, applied the test procedures on each multiply imputed data set and determined the cut points under the null scenarios that resulted in the exact and nominal level to be the same. We then used the same cut points for the non-null scenarios to determine the power of these tests.

We generated 500 data sets under the simulation scenarios described in Section 4. We also added modest and severe misspecification. The following table provides the true and imputation models used in the simulation.

[Table 1 about here.]

We calculated the number of rejections of the null hypothesis for each test in every imputed data set. Based on these empirical distributions, we established a rule based on the number data sets in which the hypothesis should rejected (under the null model) to ensure that type I error is close to the nominal level (we chose 0.05 ). For both the tests, the following two simple rules of rejecting the imputation model worked best:

1. Rule 1: Reject the imputation model if the ANOVA test is rejected in at least two of the five imputed data sets.

2. Rule 2: Reject if at least KS is rejected in at least one of the five imputed data sets or ANOVA test is rejected in at least two of the five imputed data sets.

Table 2 provides the exact level (under the null) and power (under the alternative) based on the 500 simulated data sets.

[Table 2 about here.]

The results in Table 2 suggest that though using just the ANOVA test may be sufficient to detect large departures from the true model but using both KS and ANOVA tests add considerable power to detect relatively modest differences between the true and imputation models.

\section{Conclusion}

The major emphasis of this paper is to propose a set of graphical diagnostic tools that adopts propensity score methods to assist in assessing suitability of multiple imputations for an analyst without the full knowledge of the imputation model. Additionally, the proposed diagnostics can be used by an imputer to check the validity of the imputations from the working model and refine it, if necessary. The central theme is the comparison of the imputed and observed values conditional on the estimated propensity of response even with missing values in other variables.

Two proposed diagnostic tools help to evaluate different features of imputed values. The scatter plot diagnostics assist in comparison of the conditional distributions, whereas the residual density plots are useful in comparison of means as well as second and third moments of the residual distributions. It is important to address both issues when evaluating multiple imputations. We also proposed and evaluated some simple rules for rejecting the imputation model using the significance testing framework. 


\section{Statistics}

Although, the focus was on binary and continuous variables, the method could be extended to count or semi-continuous variables. For example, in the case of semi-continuous variables, the diagnostics can be carried out in two parts. The first part uses the binary variable diagnostic tools assess the validity of imputations of zero/non-zero status. Next, conditional on the binary variable being non-zero, one can use the continuous variable approach to assess the validity of imputed continuous values. The methodology can be easily implemented in standard software packages such as R or Stata and has already been used in a complex setting [25] where they use limited techniques in the non-peer reviewed technical report [26]. This report considered mainly continuous variables, did not provide theoretical underpinnings, refinements for binary variable and the numerical tests. The present article also carries out a more thorough evaluation of the proposed tools.

The proposed approach can be used by an analyst who was not involved in the imputation process and seeks to assess congeniality of the imputation and analyst models. If the diagnostic procedures indicate problems with the imputation, then it may be prudent for the analyst to ignore the imputed values and adopt alternative approaches such as multiple imputation analysis by re-imputing the missing values in just the variables in the analyst model, the maximum likelihood or the fully Bayesian analysis. A comparison of the results with original and re-imputed values might be useful to quantify sensitivity of inferences to model misspecification. Such analysis across the data sets might be useful to assess the impact of uncongeniality on multiple imputation inferences.

There are number of limitations that can be addressed with further research. First, the approach assumes that the data set includes all the variables used in the imputation process. In some applications, the imputer may use some internally available variables and it is possible that our diagnostics may indicate problems where there are none. However, as long as all variables used in imputations are used in propensity score estimation the inferences should be valid. Second limitation is the assumption that the data are missing at random. The problems identified by the procedures may be due to nonignorable missing data mechanism rather than problems with the imputation model. The third limitation is that while focusing on variable $Y_{v}$, the estimated propensity score is constructed by averaging over imputations of all other variables $Y_{-v}$. If the models imputing those variables are severely misspecified, then the estimated propensity score may be affected. One solution is to apply the diagnostics sequentially and iteratively for all the variables until all the models show reasonable fit across all variables. Nevertheless, further work is needed to address these limitations.

\section{References}

[1] Rubin DB. Multiple Imputation for Nonresponse in Surveys. Wiley, 1987.

[2] Li KH, Raghunathan TE, Rubin D. Large sample significance levels from multiply-imputed data using momentbased statistics and an f-reference distribution. J. Am. Statist. Assoc. 1991; 86:1065-1073.

[3] Li KH, Meng X, Raghunathan T, Rubin DB. Significance levels from repeated p-values with multiply imputed data. Statist. Sinica 1991; 1:65-92.

[4] Barnard J, Rubin DB. Small-sample degrees of freedom with multiple imputation. Biometrika 1999; 86(4):948-955.

[5] X L Meng X, Rubin DB. Performing likelihood ratio tests with multiply imputed data sets. Biometrika 1995; 79:103111.

[6] Raghunathan TE, Lepkowski JM, Hoewyk JV, Solenberger P. A multivariate technique for multiply imputing missing values using a sequence of regression models. Survey methodology 2001; 27(1):85-95.

[7] Berglund P, Heeringa SH. Multiple Imputation of Missing Data Using SAS. SAS Institute, 2014.

[8] van Buuren S. Flexible Imputation of Missing Data. Chapman and Hall/CRC, 2012. 


\section{Statistics}

[9] Stata. Multiple-Imputation Reference Manual. Stata Press, 2015.

[10] Meng XL. Multiple-imputation inferences with uncongenial sources of input. Statistical Science 1994; 9(4):538-558.

[11] Kim JK, Brick JM, Fuller WA, Kalton G. On the bias of the multiple-imputation variance estimator in survey sampling. Journal of the Royal Statistical Society: Series B (Statistical Methodology) 2006; 68(3):509-521.

[12] Robins JM, Wang N. Inference for imputation estimators. Biometrika 2000; 87(1):113-124.

[13] Aboyomi K, Gelman A, Levy M. Diagnostics for multiple imputations. Applied Statistics 2008; 57(3):273-291.

[14] He Y,Zaslavsky AM, Harrington DP, Catalano P, Landrum MB. Multiple imputation in a large-scale complex survey: A practical guide. Stat Methods Med Res. 2010; 19(6):653670.

[15] Azur MJ, Stuart EA, Frangakis C, Leaf PJ. Multiple imputation by chained equations: What is it and how does it work? Int J Methods Psychiatr Res. 2011; 20(1):4049.

[16] Stuart EA, Azur M, Frangakis C, Leaf P. Multiple imputation with large data sets: A case study of the children's mental health initiative. Am J Epidemiol 2009; 169(9):11331139.

[17] Rosenbaum P, Rubin D. The central role of the propensity score in the observational studies for causal effects. Biometrika 1983; 70:41-55.

[18] Ralph B D'Agostino J, Rubin DB. Estimating and using propensity scores with partially missing data. JASA 2000; 95(451):749-759.

[19] Rosenbaum P, Rubin D. Reducing bias in observational studies using subclassification on the propensity score. Biometrika 1984; 79(387):516-524.

[20] Cochran WG, Rubin DB. Controlling bias in observational studies: A review. The Indian Journal of Statistics, Series A 1973; 35(4):417-446.

[21] Hosmer DW, Lemeshow S. Applied logistic regression. Wiley.: New York, 1989.

[22] Rubin DB. Graphical methods for assessing logistic regression models: Comment. Journal of the American Statistical Association 1984; 79:7980.

[23] Boonstra P, Bondarenko I, Park S, Voconas P, Mukherjee B. Propensity score-based diagnostics for categorical response regression models. Statistics in Medecine 2014; 33(3):455-469.

[24] Cochran W, Rubin DB. Controlling bias in observational studies: a review. Sankhy: The Indian Journal of Statistics, Series A 1973; 35:417-446.

[25] Robbins MW, Ghosh SK, Habiger JD. Imputation in high-dimensional economic data as applied to the agricultural resource management survey. Journal of the American Statistical Association 2013; 108(501):81-95.

[26] Raghunathan TE, Bondarenko I. Diagnostics for multiple impuation. SSRN Technical Report 2007; URL http: //ssrn.com/abstract $=1031750$. 


\begin{tabular}{|c|c|c|c|c|}
\hline IM & GM & Scatter plot diagnostics & Residual Density Plot & $\begin{array}{l}\text { Scatter plot of residuals vs } \\
\text { expected values }\end{array}$ \\
\hline 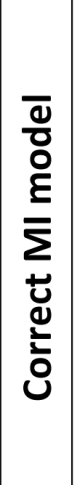 & $\begin{array}{l}\alpha_{0}=1 \\
\alpha_{1}=1 \\
\alpha_{2}=1 \\
\alpha_{3}=1 \\
\alpha_{4}=0 \\
\alpha_{5}=0\end{array}$ & 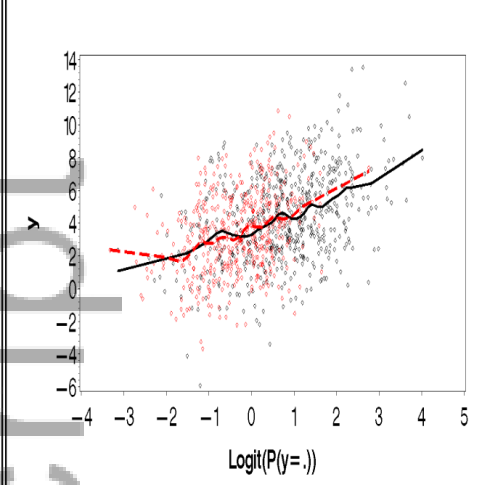 & $\begin{array}{lllllllll}0.18 \\
0.16 \\
0.14 \\
0.12 \\
0.12\end{array}$ & 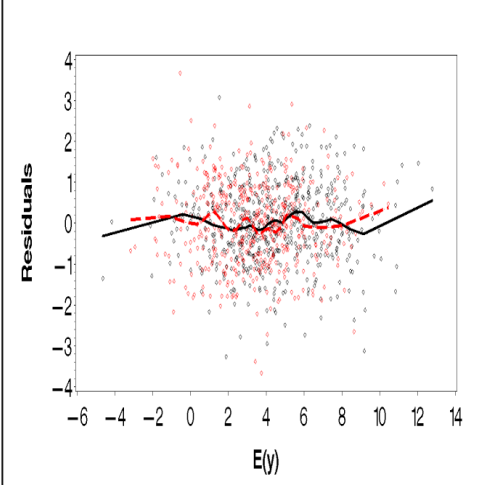 \\
\hline 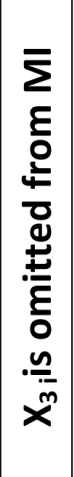 & $\begin{array}{l}\alpha_{0}=1 \\
\alpha_{1}=1 \\
\alpha_{2}=1 \\
\alpha_{3}=1 \\
\alpha_{4}=0 \\
\alpha_{5}=0\end{array}$ & 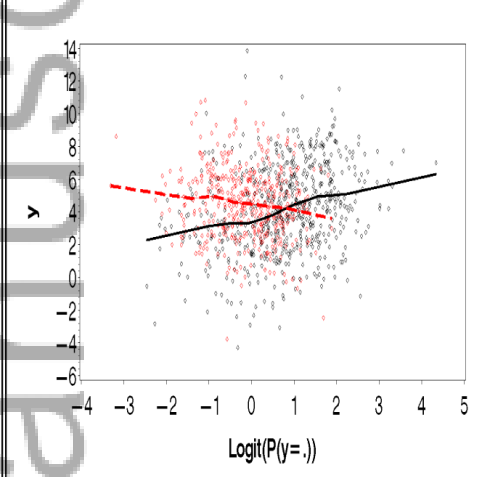 & 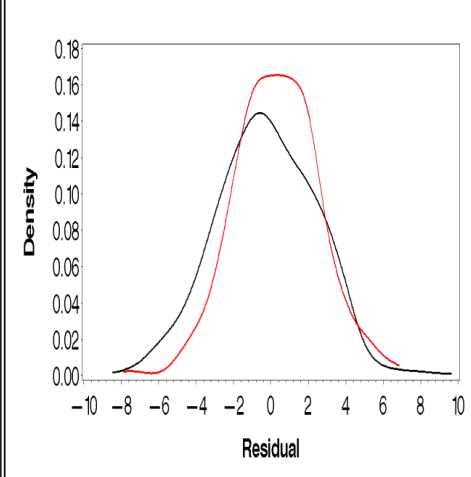 & 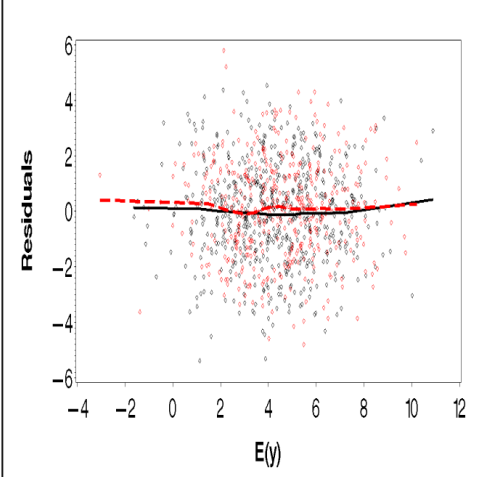 \\
\hline 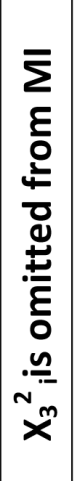 & $\begin{array}{l}\alpha_{0}=1 \\
\alpha_{1}=-1 \\
\alpha_{2}=1 \\
\alpha_{3}=1 \\
\alpha_{4}=-1 \\
\alpha_{5}=0\end{array}$ & 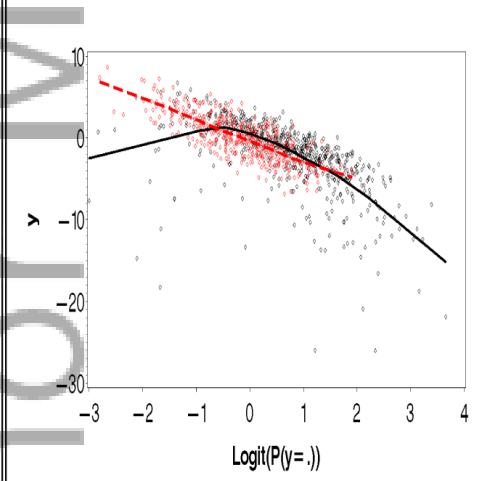 & 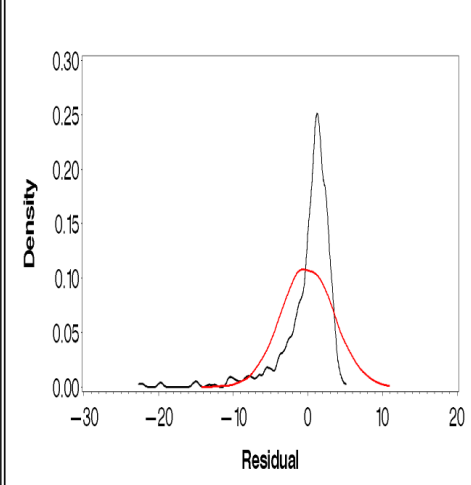 & 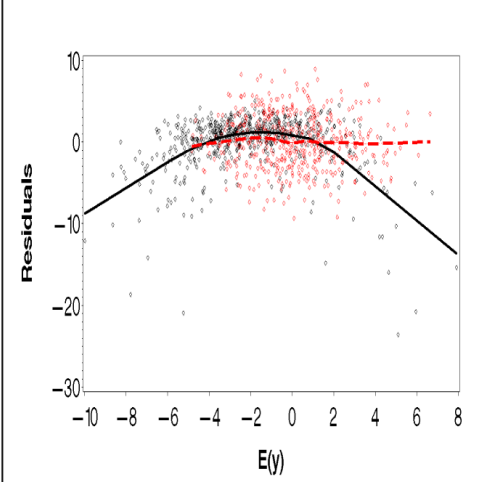 \\
\hline 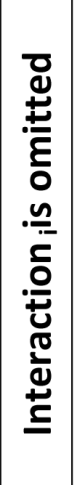 & $\begin{array}{l}\alpha_{0}=1 \\
\alpha_{1}=0 \\
\alpha_{2}=1 \\
\alpha_{3}=1 \\
\alpha_{4}=0 \\
\alpha_{5}=.5\end{array}$ & 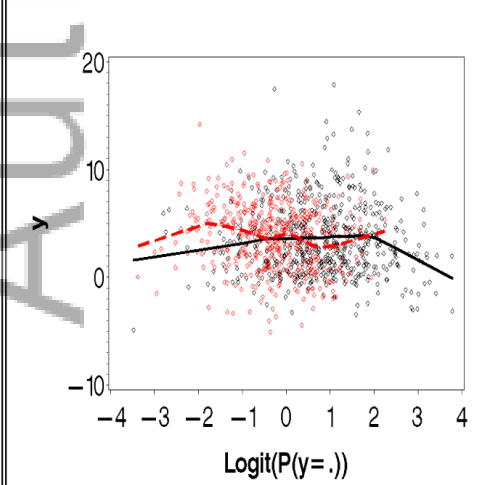 & \begin{tabular}{r|r}
0.16 \\
0.14 \\
0.12 \\
0.10 \\
0.08 \\
0.06 \\
0.04 \\
0.02 \\
0.00 \\
0 \\
0
\end{tabular} & 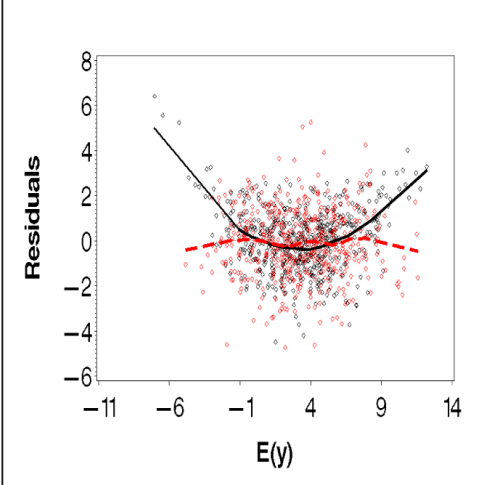 \\
\hline
\end{tabular}

Figure 1. Diagnostics for continuous variables. 


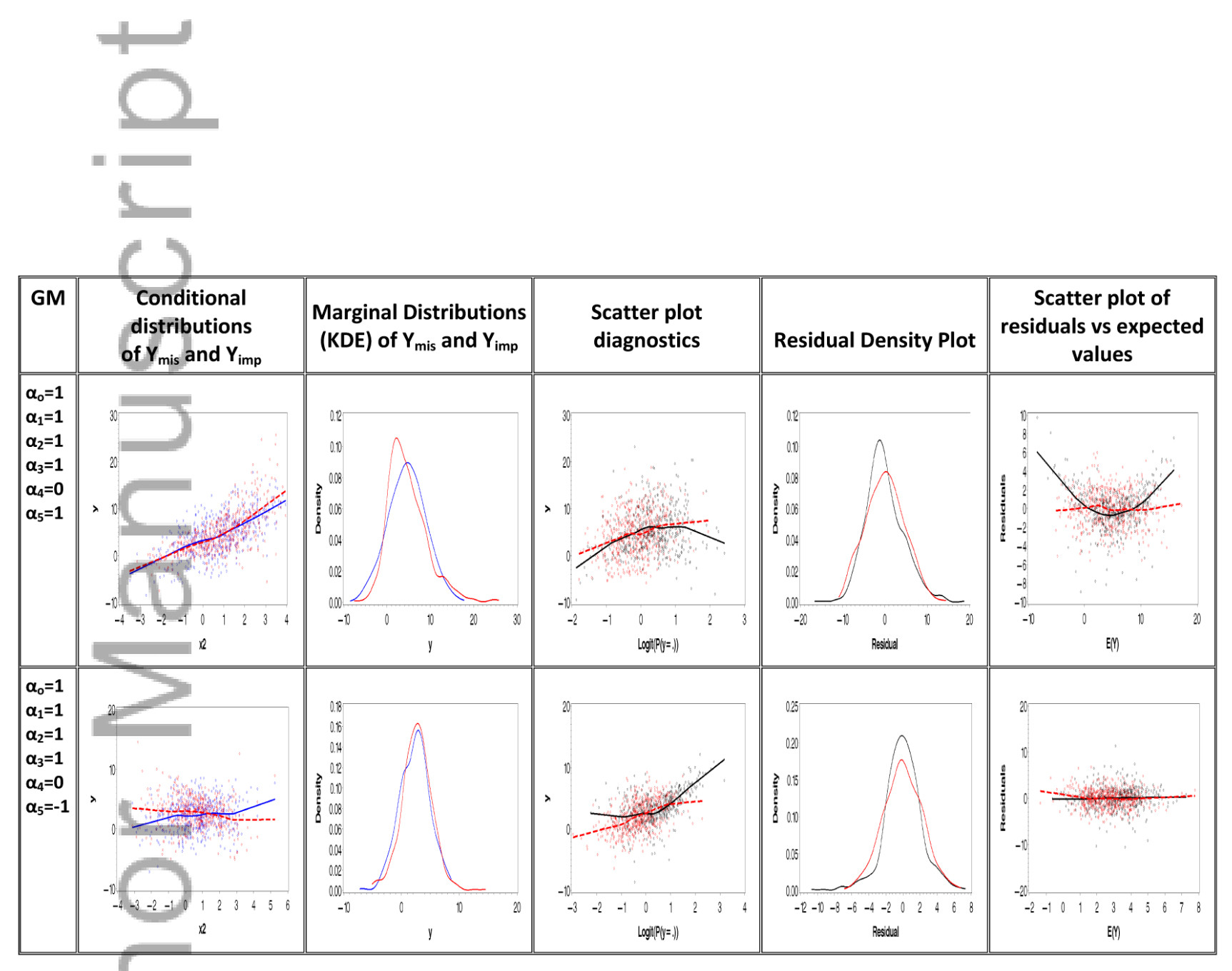

Figure 2. Targets for Scatter Plot and residual Density Diagnostics. 


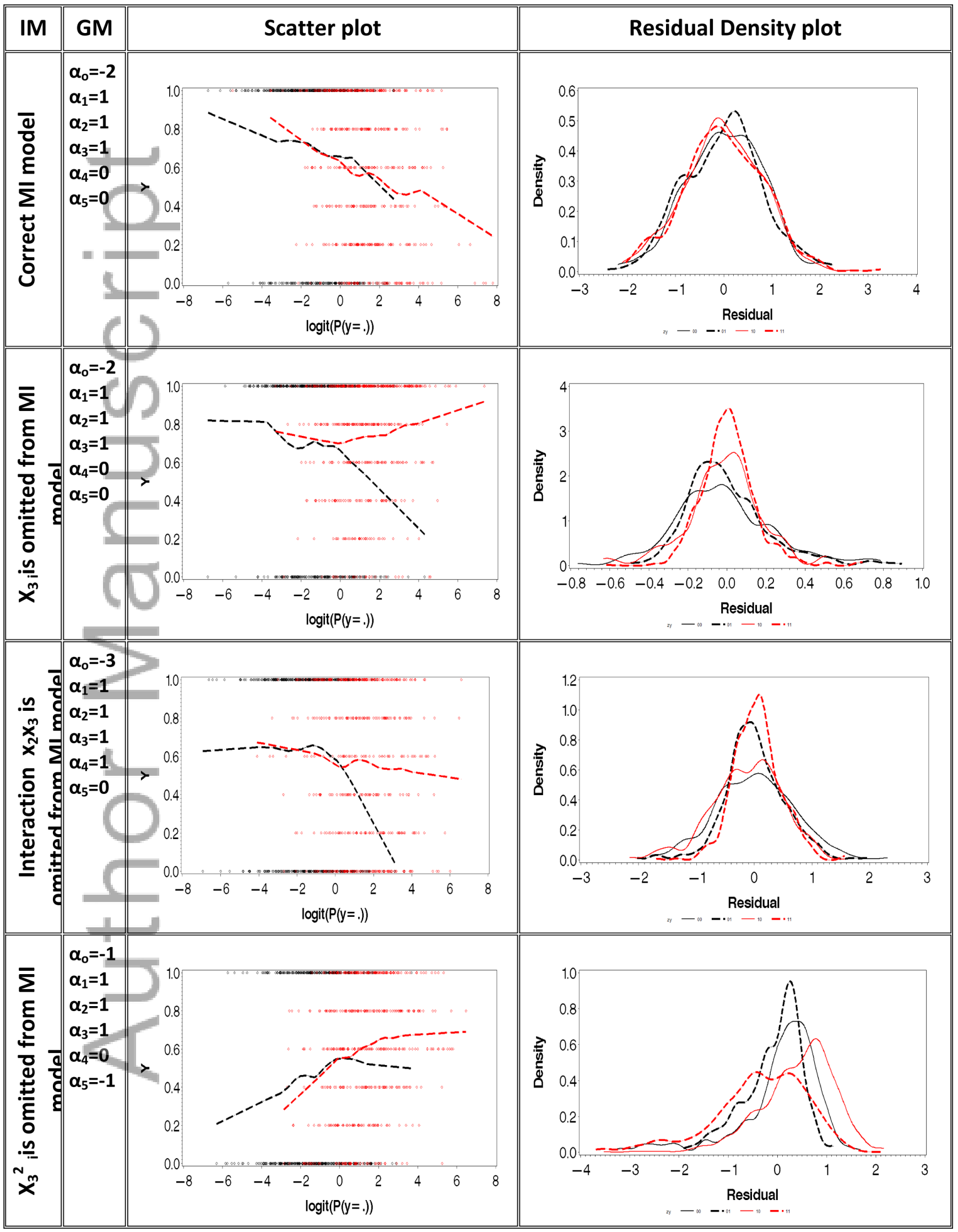

Figure 3. Diagnostics for Binary variables. 


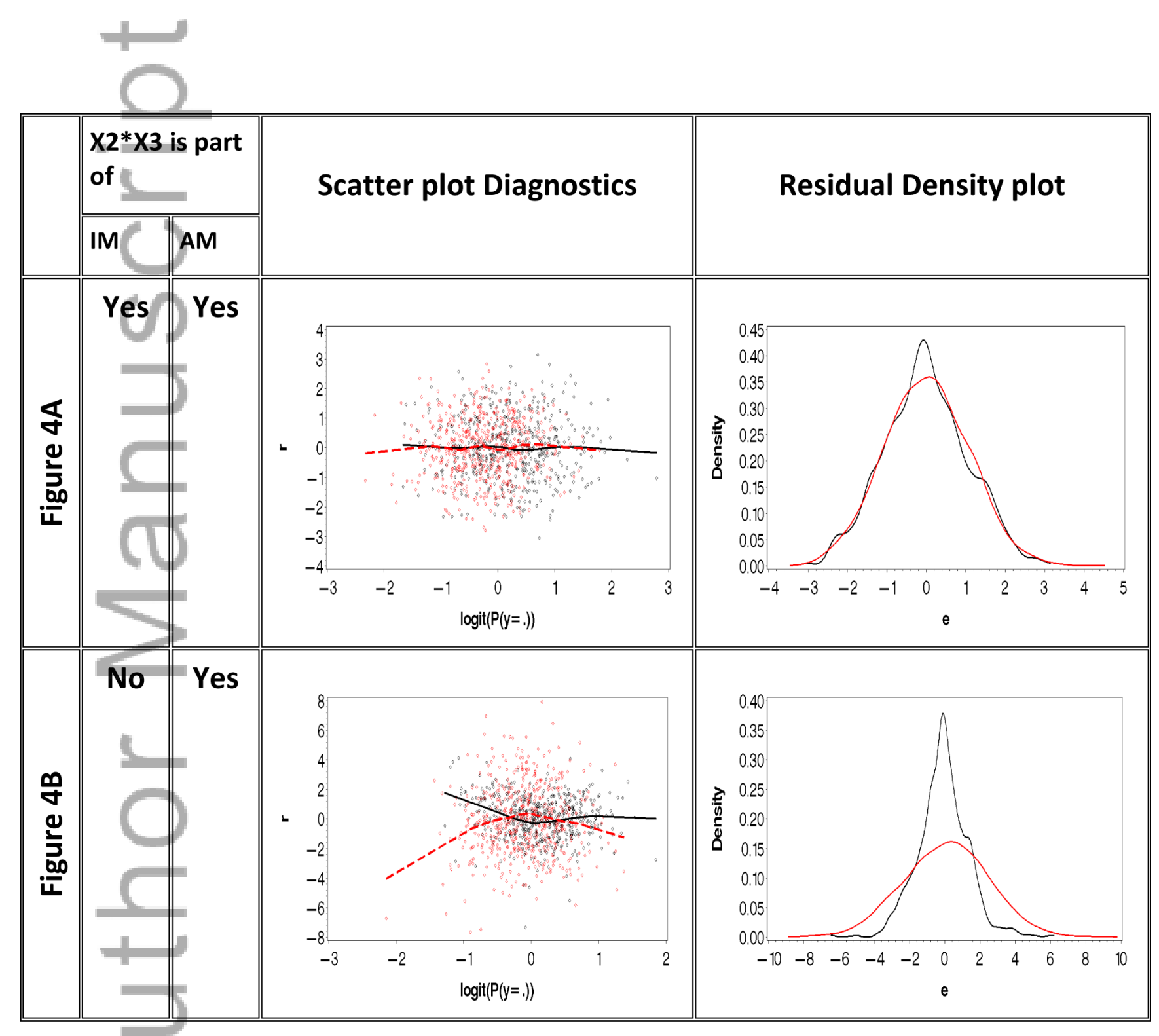

Figure 4. Use of the Scatter Plot and Residual Density Diagnostic to check Congeniality. 

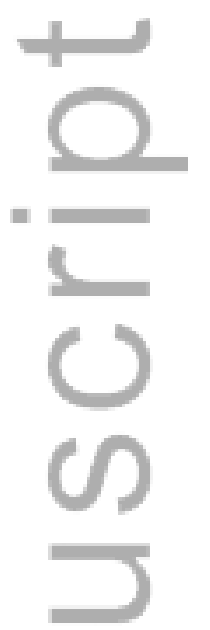

Table 1. True and Imputation models used in determining the exact and power of ANOVA and KS procedures. Residual variance is 1 for all seven true models

\begin{tabular}{|c|c|c|c|}
\hline Model Number & Type of Model & True Model & Imputation Model \\
\hline 1 & Null & $E(y)=1+x_{1}+x_{2}+x_{3}$ & $y \sim N\left(\alpha_{o}+\alpha_{1} x_{1}+\alpha_{2} x_{2}+\alpha_{3} x_{3}, \sigma^{2}\right)$ \\
\hline 2 & Omitted & $E(y)=1+x_{1}+x_{2}+x_{3}$ & $y \sim N\left(\alpha_{o}+\alpha_{1} X_{1}+\alpha_{2} x_{2}, \sigma^{2}\right)$ \\
3 & variable & $E(y)=1+x_{1}+x_{2}+0.5 x_{3}$ & Same as above \\
\hline 4 & Interaction & $E(y)=1+x_{1}+x_{2}+x_{3}+x_{2} x_{3}$ & $y \sim N\left(\alpha_{o}+\alpha_{1} x_{1}+\alpha_{2} x_{2}+\alpha_{2} x_{3}, \sigma^{2}\right)$ \\
5 & omitted & $E(y)=1+x_{1}+x_{2}+x_{3}+0.5 x_{2} x_{3}$ & same as above \\
\hline 6 & Square term & $E(y)=1+x_{1}+x_{2}+x_{3}+x_{3}^{2}$ & $y \sim N\left(\alpha_{o}+\alpha_{1} x_{1}+\alpha_{2} x_{2}+\alpha_{3} x_{3}, \sigma^{2}\right)$ \\
7 & omitted & $E(y)=1+x_{1}+x_{2}+x_{3}+0.5 x_{3}^{2}$ & Same as above \\
\hline
\end{tabular}




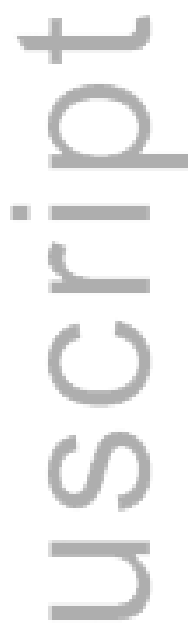

Table 2. The exact level and power (both in \%) based on the two rules for rejecting the imputation model

\begin{tabular}{|c|c|c|}
\hline Model & Rule 1 & Rule 2 \\
\hline 1 & 5.2 & 6.2 \\
2 & 98.8 & 99.4 \\
3 & 50.4 & 64.4 \\
4 & 37.8 & 87.6 \\
5 & 36.4 & 72.8 \\
6 & 86.4 & 100 \\
7 & 13.8 & 72.2 \\
\hline
\end{tabular}

